\title{
Control of Neoclassical Tearing Modes by Sawtooth Control
}

\author{
O. Sauter, ${ }^{1}$ E. Westerhof, ${ }^{2}$ M. L. Mayoral, ${ }^{3,4}$ B. Alper, ${ }^{3}$ P. A. Belo, ${ }^{5}$ R. J. Buttery, ${ }^{3}$ A. Gondhalekar, ${ }^{3}$ T. Hellsten, ${ }^{6,7}$ \\ T. C. Hender, ${ }^{3}$ D. F. Howell, ${ }^{3}$ T. Johnson, ${ }^{6}$ P. Lamalle,,${ }^{8,7}$ M. J. Mantsinen, ${ }^{9}$ F. Milani, ${ }^{3}$ M. F. F. Nave, ${ }^{5}$ F. Nguyen, ${ }^{4}$ \\ A. L. Pecquet, ${ }^{4}$ S. D. Pinches, ${ }^{10}$ S. Podda, ${ }^{11}$ and J. Rapp ${ }^{12}$ \\ ${ }^{1}$ Centre de Recherches en Physique des Plasmas, Ass. EURATOM-Confédération Suisse, EPFL, 1015 Lausanne, Switzerland \\ ${ }^{2}$ FOM-Rijnhuizen, Ass. EURATOM-FOM, TEC, 3430 BE Nieuwegein, The Netherlands \\ ${ }^{3}$ EURATOM/UKAEA Fusion Ass., Abingdon, United Kingdom \\ ${ }^{4}$ DRFC, CEA Cadarache, Ass. EURATOM/CEA, France \\ ${ }^{5}$ Centro de Fusao Nuclear, Instituto Superior Tecnico, Lisboa, Portugal \\ ${ }^{6}$ Association EURATOM-NFR, Royal Institute of Technology, Stockholm, Sweden \\ ${ }^{7}$ EFDA-JET Close Support Unit, Abingdon, United Kingdom \\ ${ }^{8}$ LPP/ERM-KMS, Ass. EURATOM-Belgian State, TEC, Brussels, Belgium \\ ${ }^{9}$ Helsinki University of Technology, Ass. EURATOM-Tekes, Finland \\ ${ }^{10}$ Max-Planck-IPP, IPP-EURATOM Ass., Garching, Germany \\ ${ }^{11}$ CRF, Ass. EURATOM-ENEA sulla Fusione, Frascati, Italy \\ ${ }^{12}$ Institut für Plasmaphysik, EURATOM Ass., TEC, Jülich, Germany
}

(Received 14 August 2001; published 25 February 2002)

\begin{abstract}
The onset of a neoclassical tearing mode (NTM) depends on the existence of a large enough seed island. It is shown in the Joint European Torus that NTMs can be readily destabilized by long-period sawteeth, such as obtained by sawtooth stabilization from ion-cyclotron heating or current drive. This has important implications for burning plasma scenarios, as alpha particles strongly stabilize the sawteeth. It is also shown that, by adding heating and current drive just outside the inversion radius, sawteeth are destabilized, resulting in shorter sawtooth periods and larger beta values being obtained without NTMs.
\end{abstract}

PACS numbers: 52.55.Fa, 52.35.Py, 52.50.Gj

One of the main objectives of fusion plasma physics research is to optimize $\beta=p /\left(B_{0}^{2} / 2 \mu_{0}\right)$, the ratio of the plasma pressure to the magnetic pressure, where $B_{0}$ is the main confining magnetic field. In tokamak discharges, the maximum achievable $\beta$ is limited by macroscopic unstable modes well described by the ideal magnetohydrodynamic fluid theory. This limit is relatively high in standard high confinement scenarios ( $H$-modes), with a monotonic safety factor $q$. The value of $q$ is determined by the ratio of toroidal to poloidal turns of the magnetic field line. It has been found in the past few years $[1,2]$ that pressure driven modes can be destabilized at $\beta$ values much lower than the ideal limit. These neoclassical tearing modes (NTMs) form islands near resonant surfaces corresponding to rational $q$ values, in particular the $m=3 / n=2$ and $2 / 1$ surfaces, where $m$ and $n$ are the poloidal and toroidal mode numbers. Once a seed island is formed and is sufficiently wide to locally flatten the density and/or temperature profile, it reduces the self-generated bootstrap current which further destabilizes the mode [1]. The main detrimental effect of these modes is a loss of energy and particle confinement which can significantly reduce both the central temperature and density and may thus adversely affect the performance in the baseline scenario, envisaged for reactorlike plasmas [3].

It is well known both theoretically and experimentally that a finite seed island is required before the island can be further destabilized by the perturbed bootstrap current [2]. This is due to stabilizing terms which are im- portant at small island size, typically when the island is of the order of the ion poloidal Larmor radius $(\approx 2 \mathrm{~cm})$. The trigger mechanism for this seed island is usually associated with a sawtooth crash or its pre- or postcursor, but can be due to other perturbations. Therefore a possible way to avoid NTMs is to keep the seed islands smaller than the critical seed island width. This has been tested indirectly using scenarios with $q>1$, such as to avoid the main internal perturbations, the sawtooth activity [4]. However, these scenarios are inherently transient as it would require too much current drive to keep $q>1$ in the center of a standard scenario in a reactorlike tokamak. A new and better scenario would be to maintain the sawtooth activity, which is deemed useful in a reactor for avoiding ash accumulation in the center, but at a level such that the seed islands induced at $q=1.5$ and 2 are small. In this Letter, we first demonstrate that by controlling the sawtooth period $\left(\tau_{\text {saw }}\right)$, due to sawtooth stabilization, the $\beta$ values at which NTMs are observed can be changed by more than a factor of 2 . Then it is shown how the performance can be increased by destabilizing the sawteeth, keeping $\tau_{\text {saw }}$ short. Therefore a new scheme is proposed for $H$-mode burning plasmas: prevent NTMs by controlling the main perturbations causing the seed islands.

In the Joint European Torus (JET), sawteeth can be affected by waves in the ion cyclotron range of frequencies (ICRF). Using a fundamental $H$ minority scheme, a large population of fast ions is created, which has a stabilizing effect on the internal kink mode and leads to long $\tau_{\text {saw }}$ 
[5(a)]. Moreover, depending on the ICRF antenna phasing, the fast particle pressure inside $q=1$ and thereby $\tau_{\text {saw }}$ can be differently modified due to the ICRF-induced pinch of the resonating trapped ions [5(b)]. In recent experiments, this mechanism has been utilized with $4.5 \mathrm{MW}$ of ICRF power applied at a frequency of $42 \mathrm{MHz}$ (Fig. 1). The discharge with $+90^{\circ}$ phasing (cocurrent, dark line), leads to longer $\tau_{\text {saw }}, 630 \mathrm{~ms}$, than the discharge with $-90^{\circ}$ phasing (countercurrent, grey line), $200 \mathrm{~ms}$. As the resonance layer is positioned near the sawtooth inversion radius $\left(R_{\text {inv }}\right)$, the Ion Cyclotron Current Drive (ICCD), which can modify locally the current profile and consequently destabilize or stabilize sawteeth, also plays a role [5(c),6(a),6(b)]. In addition, for the discharges shown in Fig. 1a, a similar ramp of neutral beam injection (NBI) power is imposed in order to determine the onset beta value of the $3 / 2$ NTM mode (Fig. 1b). The ICRF and NBI power, normalized beta $\left(\beta_{N}\right)$, soft x-ray (SXR) emission, and neutron rates are well matched, that is, the profiles are similar. However, at $16.5 \mathrm{~s}$ a $3 / 2$ NTM is destabilized in the discharge which has long sawtooth periods. The mode is triggered exactly at the sawtooth crash, at $\beta_{N}=1, P_{\mathrm{NBI}}=5 \mathrm{MW}$ and $P_{\mathrm{RF}}=4.5 \mathrm{MW}$. On the other hand, the case with shorter sawtooth periods does not trigger NTMs even at full power, $\beta_{N} \approx 2, P_{\mathrm{NBI}}=16 \mathrm{MW}$, and $P_{\mathrm{RF}}=4.5 \mathrm{MW}$. $\tau_{\text {saw }}$ can be controlled by moving the fundamental $H$ resonance position. In this way, by increasing $\tau_{\text {saw }}$ from 240 to $720 \mathrm{~ms}$, changing $B_{0}$ from 2.23 to $2.6 \mathrm{~T}$, with $+90^{\circ}$ phasing, while keeping the $q$ profile fixed, it was shown that NTMs are destabilized at low $\beta_{N}(\sim 1)$ when
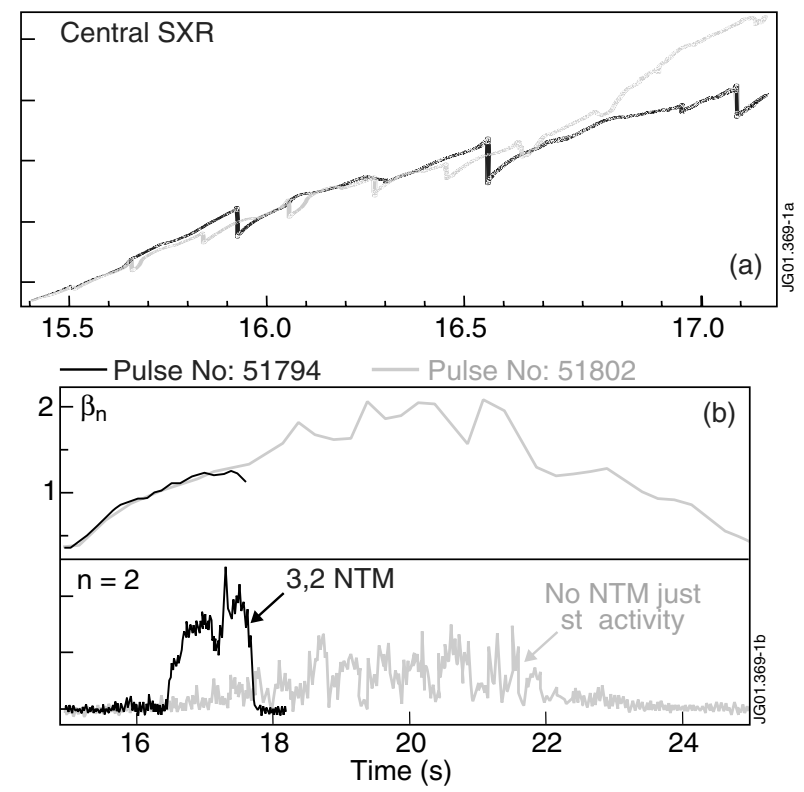

FIG. 1. Two similar discharges with $2.4 \mathrm{~T}, 2.4 \mathrm{MA}, 4.5 \mathrm{MW}$ ICRF, same $P_{\mathrm{NBI}}$ ramp. Only the antenna phasing has been changed: $+90^{\circ}$, No. 51794 (long ST, dark line) and $-90^{\circ}$, No. 51802 (short ST, grey line). (a) SXR emission. (b) $\beta_{N}$ and $n=2$ MHD activity. In No. 51794, a NTM is triggered at the first crash when $\beta_{N}>\beta_{N \text { marg }}$. $\tau_{\text {saw }} \geq 600 \mathrm{~ms}$ at this medium field [7]. Note that these modes were actually already observed and described as a consequence of sawtooth stabilization in 1988 [5(a)]. This also explains why discharges dominantly heated by ICRF have much lower $\beta_{N \text { onset }}$ than NBI only discharges. In some NBI only cases, with the power ramp creating a long first sawtooth period, NTMs can also be destabilized at low beta at the crash. These experiments demonstrate the direct effect of controlling the sawtooth activity on the NTM onset. In previous standard JET experiments, NTMs were never destabilized even at full NBI power (17 MW) for $I_{p}>1.7 \mathrm{MA}, B_{0}>1.7 \mathrm{~T}$. This was thought to be due to lack of power. However, through sawtooth control, NTMs can now be triggered at low $\beta_{N}$. As a further test, $5 \mathrm{MW}+90^{\circ}$ ICRF was added to a high magnetic field discharge with $3.3 \mathrm{~T}, 3.3 \mathrm{MA}$, and 5.4 MW NBI power. A $1.6 \mathrm{~s}$ sawtooth free period is induced, after which crash a $3 / 2$ NTM is triggered at $18.74 \mathrm{~s}$. Figure 2 shows that a large seed island of $6 \mathrm{~cm}$ is directly triggered, close to the saturated width at this low $\beta$, thus the island width remains almost constant. Figure 2 also shows that although the precursor $n=1$ is large around $15-17 \mathrm{~s}$ no mode is triggered while the crash at $18.74 \mathrm{~s}$ has no significant precursor activity. Similarly the crash of the central chord SXR at this sawtooth is not much larger than at the previous crash. The only correlation observed so far in these experiments is the sawtooth period itself. However, there is no linear correlation with the seed island triggered. Further experimental and theoretical studies are necessary to quantify this relationship.

The direct link between sawtooth stabilization and low onset $\beta$ is confirmed by the strong correlation between the

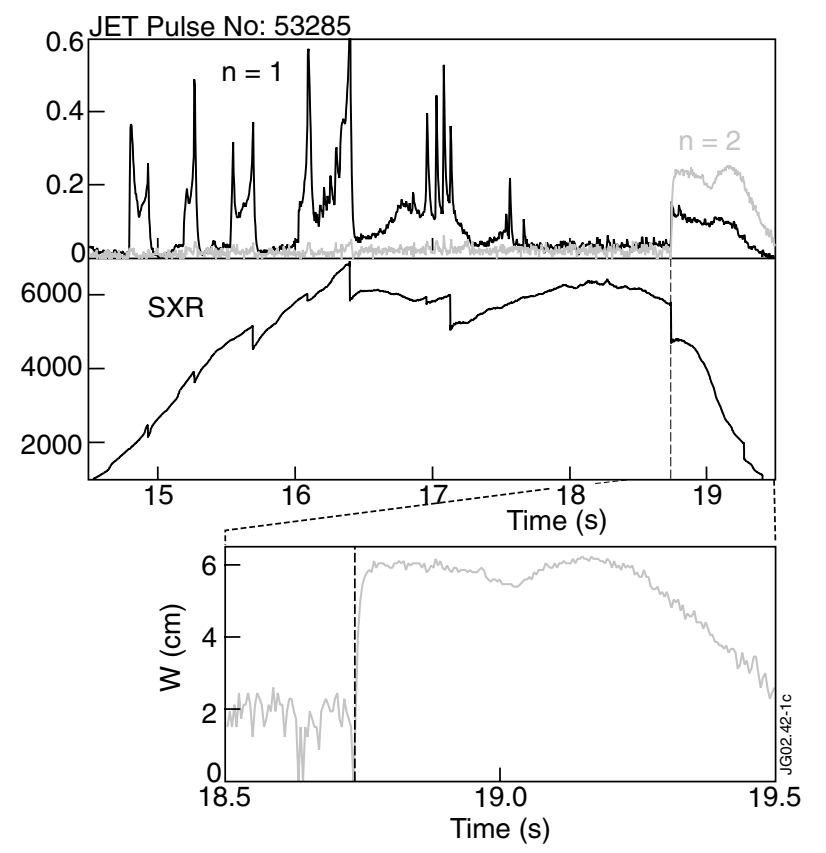

FIG. 2. High field case, $3.3 \mathrm{~T}, 3.3 \mathrm{MA}, \beta_{N} \approx 1$. The $3 / 2$ mode is triggered at the crash after a $1.6 \mathrm{~s}$ sawtooth period. The island width is directly formed at about $6 \mathrm{~cm}$. 
sawtooth crash time and the NTM onset, as opposed to cases with short sawtooth periods [8]. This is the main result of this paper and is particularly important for burning plasma discharges: alpha particles are predicted to strongly stabilize the sawteeth [9]. Using a model based on Ref. [9], we are able to simulate the effect of fast particles [10] and of localized current drive (CD) and electron power deposition, as shown in detailed experiments in TCV (tokamak configuration variable) [11]. However, it is out of the scope of this paper to quantify the amount of local counter-CD needed to significantly reduce $\tau_{\text {saw }}$ in such scenarios. In addition, as the exact physical mechanism by which the seed island is formed during the crash is not known, it is not possible to predict what is a short enough sawtooth period. However, we have already tested if beta onset can be increased in the present discharges using scenarios aimed at destabilizing the sawteeth. This is a more difficult task, since in present low field discharges the $\tau_{\text {saw }}$ are of medium size, about $200-300 \mathrm{~ms}$, and the sawtooth crash is only one of the trigger mechanisms $[2,8]$.

A reference discharge is chosen for which NTMs are destabilized with NBI only. This is achieved by choosing a low magnetic field case (1.2-1.4 T), which requires the use of second harmonic $H$ minority ICRF to drive localized current (ICCD) and affect the sawteeth. Scanning the resonance position across $R_{\text {inv }}$ shows that $\tau_{\text {saw }}$ has a minimum when the resonance position is just outside $R_{\text {inv }}$ [6]. Using this method the magnetic field $\left(B_{0}=\right.$ $\left.1.2 \mathrm{~T}, I_{p}=1.2 \mathrm{MA}\right)$ needed to couple at this position on the high field side (HFS) can be found. This is exploited in the results shown in Fig. 3, where two cases with and without ICRF are compared, but with the same total power waveform. The NBI-only discharge, 52712, triggers a $3 / 2 \mathrm{NTM}$ at $\beta_{N} \approx 2.7, P_{\text {tot }}=8 \mathrm{MW}$ (typical for this field and current), much earlier than the one with ICRF which reaches a maximum $\beta_{N} \approx 3.8$ without $3 / 2$ modes. Actually a $5 / 4$ mode is triggered at $t=25.0 \mathrm{~s}$, then a $4 / 3$ mode at $26.0 \mathrm{~s}$, and finally a $3 / 2$ mode at $t=26.2 \mathrm{~s}, \beta_{N} \approx 3.6$ in the ICRF case. Note that during the high $\beta$ phase the local magnetic field decreases due to diamagnetic effects and the resonance position moves away from the optimum position for sawtooth destabilization, as will be discussed later. Also fishbones are usually present when $\beta_{N} \geq 3$ [8], so it is not clear if sawtooth activity or fishbones have triggered these NTMs. However, it should be noted that the discharge is very close to the ideal beta limit, $\beta_{N} \approx 4 l_{i} \approx 4$, and therefore much higher than the value at which a stationary standard $H$-mode would be in burning plasmas [3].

The peculiar time evolution of the island width and the related confinement degradation needs clarification. While the NBI power is further increased (Fig. 3), the island width decreases at about $24.5 \mathrm{~s}$ and saturates to a lower value. Consequently the confinement degradation is reduced and $\beta_{N}$ recovers, reaching similar values to the other case which has no 3/2 NTM (about 25 s). This effect is termed "self-healing" and occurs as the NBI power

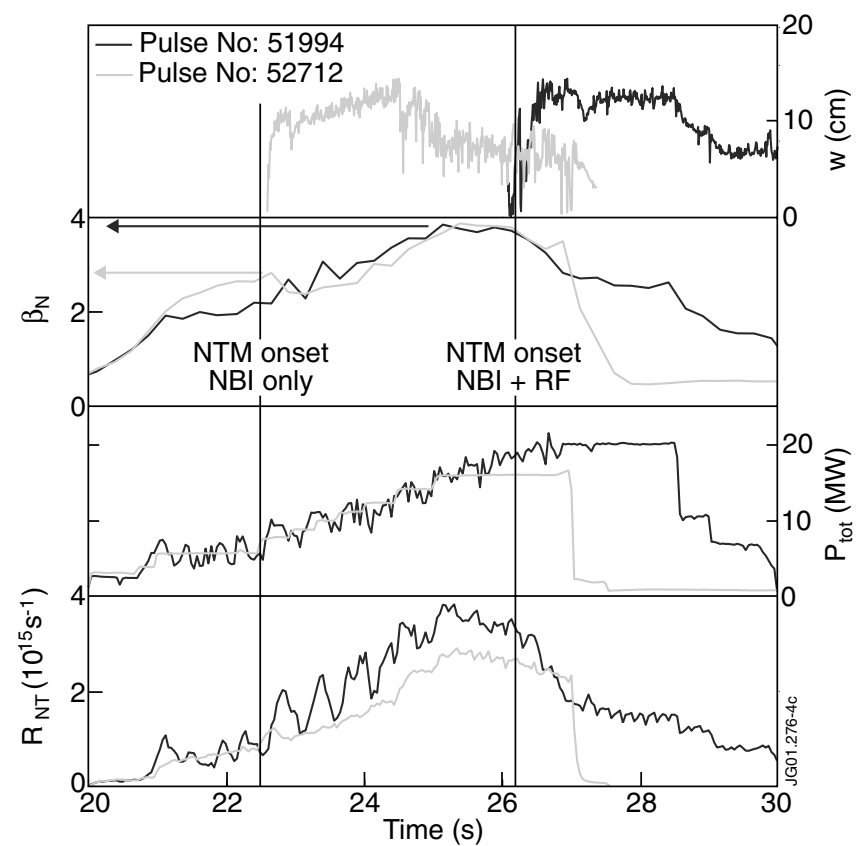

FIG. 3. Low field case, 1.2 T, 1.2 MA. Two discharges with same total power waveform, one with NBI heating only, 52712 (grey line), and one with NBI + ICRF (4.5 MW) heating with the resonance position just outside $R_{\text {inv }}, 51994$ (dark line). The island width $w, \beta_{N}$, total power and neutron rate are shown.

increases (at $P_{\mathrm{NBI}} \approx 15 \mathrm{MW}, \beta_{N} \approx 3.3$ ). It is probably linked to the increased rotation with increased NBI power, as confirmed by a rapid mode frequency increase at $24.5 \mathrm{~s}$, enabling this self-healing and the saturated width to settle at a lower island size. However, the neutron rate does not fully recover, due to its higher sensitivity to central plasma parameters and the presence of the $3 / 2$ mode, albeit at a small amplitude.

The sawtooth period near $t=22 \mathrm{~s}$ in Fig. 3 is smaller for the NBI + ICRF case, $130 \mathrm{~ms}$, than for the NBI case, $300 \mathrm{~ms}$. Since NBI fast particles also stabilize sawteeth [10], we have repeated the case 52712 but with the same NBI power waveform as 51994 instead of the same total power. In this case a NTM is triggered at $\beta_{N} \approx 2.1$ and $\tau_{\text {saw }}=180 \mathrm{~ms}$. This comparison with the same NBI or the same full power is important as rotation also plays an important role. In particular a smaller differential rotation between $q=1$ and $q=1.5$ surfaces leads to lower $\beta_{N \text { onset }}$ [12]. This can contribute to the lower $\beta_{N \text { onset }}$ values usually observed in ICRF dominated scenarios. Therefore, it is only by a careful choice of the resonance position, such as to destabilize the sawteeth, that we have been able to actually increase $\beta_{N \text { onset }}$ with the application of ICRF. This is also well illustrated in Fig. 4, where a detailed shot-toshot magnetic field scan with constant $q$ profile has been performed in order to find the best resonance position. Two groups of discharges can be identified: three shots which have no NTMs up to $\beta_{N} \approx 3.6$, similar to the HFS case 51994 , and in the other discharges a $3 / 2$ NTM is triggered at $\beta_{N} \approx 3$ or lower. From the time evolution of the resonance position, the first group corresponds to cases for 


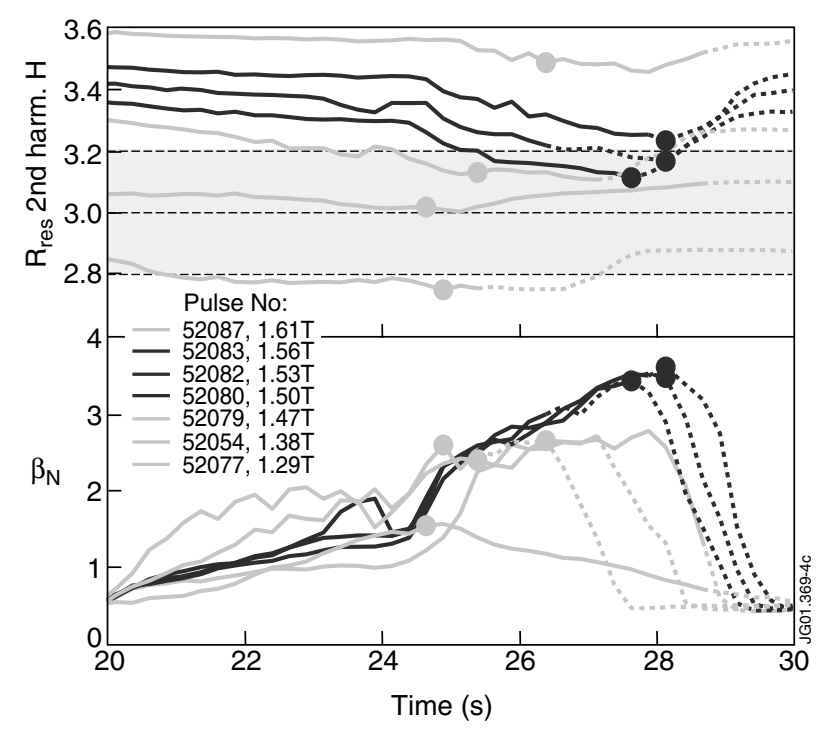

FIG. 4. Resonance position and $\beta_{N}$ for discharges with $B_{0}$ changed keeping with $I_{p} / B_{0}$ constant. The dots mark the NTMs onset and the dashed portions when $P_{\text {ICRF }}<1$ MW (bad coupling). The shaded area sketches the region inside $R_{\text {inv }}$.

which the resonance is just outside $R_{\text {inv }}$ during the ramp. $\tau_{\text {saw }}$ cannot be determined in $H$ modes, because of ELMs activity and fishbones, but $\tau_{\text {saw }}$ is minimum for 52083 $(180 \mathrm{~ms})$ and maximum farther inside $52079(300 \mathrm{~ms})$ at $23.5 \mathrm{~s}$. The width of this "good" region is about $20 \mathrm{~cm}$, and the position corresponds to the region where the minimum $\tau_{\text {saw }}$ is found, as mentioned earlier [6]. The width in magnetic field, $\Delta B_{0} \approx 0.1 \mathrm{~T}$, confirms that an accurate control is needed, in agreement with the simulations of the localized ICCD profiles [6(c)]. The sensitivity presented in Fig. 4 corresponds to the effect of localized current drive and electron heating as observed in detailed experiments on TCV [11]. This confirms the relation to the control of the sawteeth.

In conclusion, we have clearly demonstrated experimentally the direct relation between sawteeth and NTM onset and thereby the possible control of NTMs by control of the sawtooth period. We have shown that by increasing $\tau_{\text {saw }}, 3 / 2$ NTMs are triggered at the sawtooth crash, at very low $\beta_{N}$ values down to $\beta_{N \text { marg }} \approx 0.5-1$. This has important implications for burning plasmas which are predicted to have long sawtooth periods due to strong stabilization by alpha particles. The dependence of $\beta_{N \text { onset }}$ on the period is not linear and the critical value is at about $600 \mathrm{~ms}$ at $B \approx 2.4-2.8 \mathrm{~T}$ in JET. Further detailed analyses and experiments are needed to quantify and understand this effect. A possible explanation is that a longer $\tau_{\text {saw }}$ allows the shear at $q=1$ to become larger at the crash [10], leading to a larger magnetic perturbation due to the reconnection process. As $\beta_{N \text { marg }}$ is low in standard JET $H$-modes [7], it is only by controlling the maximum size of the seed islands that NTMs can be avoided. This has been further confirmed as $\beta_{N}$ values close to the ideal limit have been reached before triggering NTMs by accurately positioning the ICRF resonance such as to destabilize the sawteeth. Sawteeth are useful for avoiding impurity accumulation, therefore sawteeth destabilization is the most promising scenario. The following step is to demonstrate that sawteeth can be controlled even with a large stabilizing contribution from fast particles, such as alpha particles resulting from the fusion reactions [9]. Localized ICCD or counter electron cyclotron $\mathrm{CD}$, of the order of $1 \%-2 \%$ of the plasma current [11], should enable a reduction in the sawtooth period.

We are grateful to the UKAEA for the efficient operation of the JET and contributors to the EFDA-JET Workprogramme [13]. This work was performed under the European Fusion Development Agreement and was supported in part by the Swiss National Science Foundation, EURATOM, and the UK Department of Trade and Industry.

[1] O. Sauter et al., Phys. Plasmas 4, 1654 (1997).

[2] R. J. Buttery et al., Plasma Phys. Controlled Fusion 42, B61 (2000), and references therein.

[3] ITER Physics Basis, Nucl. Fusion 39, 2137 (1999).

[4] R. J. La Haye et al., Nucl. Fusion 40, 53 (2000).

[5] (a) D. J. Campbell et al., Phys. Rev. Lett. 60, 2148 (1988); (b) L.-G. Eriksson et al., Phys. Rev. Lett. 81, 1231 (1998); (c) V.P. Bhatnagar et al., Nucl. Fusion 34, 1579 (1994).

[6] (a) M.-L. Mayoral et al., in Proceedings of the 14th Topical Conference on RF Power in Plasmas, Oxnard, 2001 (to be published); (b) F. Nguyen et al., in Proceedings of the 28th EPS Conference on Controlled Fusion and Plasma Physics, Portugal, 2001 (Institute of Physics, Bristol, 2001), p. 2.081; (c) M. Mantsinen et al., ibid., p. 2.083; (d) (to be published).

[7] O. Sauter et al., in Proceedings of the 28th EPS Conference on Controlled Fusion and Plasma Physics, Portugal, 2001, European Physical Society Conference Abstract (Institute of Physics, Bristol, 2001), Vol. 25, p. 5.001; http://epsppd. epfl.ch

[8] P. Belo et al., in Proceedings of the 28th EPS Conference on Controlled Fusion and Plasma Physics, Portugal, 2001, European Physical Society Conference Abstract (Ref. [7]), p. 5.004 .

[9] F. Porcelli et al., Plasma Phys. Controlled Fusion 38, 2163 (1996).

[10] C. Angioni et al., Plasma Phys. Controlled Fusion (to be published).

[11] O. Sauter et al., Phys. Plasmas 8, 2199 (2001).

[12] R. J. Buttery et al., in Proceedings of the 28th EPS Conference on Controlled Fusion and Plasma Physics, Portugal, 2001, European Physical Society Conference Abstract (Ref. [7]), p. 5.011.

[13] See J. Pamela et al., in Proceedings of the 18th IAEA Fusion Energy Conference, Sorrento, 2000 (IAEA, Vienna, 2001). 